\title{
Posicionamiento de las empresas Hondureñas en jóvenes de San Pedro Sula mediante redes sociales ${ }^{1}$
}

\author{
Andrea Cecilia Vallecillo Moreno ${ }^{2}$ \\ Mario Raúl Martínez Aguilar \\ UNITEC, San Pedro Sula, Honduras.
}

(Recibido: Diciembre, 2014 y Aceptado: Junio, 2015)

\begin{abstract}
Resumen:
Esta investigación indaga el comportamiento de jóvenes sampedranos respecto al uso de redes sociales y el posicionamiento de empresas hondureñas cuyos servicios o productos se dedican a ello, con el objetivo de entregar a las empresas información para mejorar su acceso al mercado juvenil. Los resultados de la investigación provienen de la aplicación de una encuesta a trescientas ochenta y cuatro personas entre las edades de quince y veinticuatro años durante el mes de septiembre, con una confiabilidad basada en el Alfa de Cronbach de 0.799. La mayoría de los jóvenes sampedranos utilizan medios electrónicos no tradicionales, tienen acceso a internet todo el tiempo, utilizan redes sociales y están dispuestos a recibir publicidad mediante ellas. Sin embargo, es muy escaso el conocimiento de los jóvenes acerca de cuáles son las empresas hondureñas cuyos servicios o productos son dedicados a ellos, y más bajo aún; de cuáles son las empresas hondureñas que están interactuando mediante redes sociales con ellos. El presente estudio contiene información que contribuirá a que tenga éxito la publicad de las empresas hondureñas dirigidas al mercado juvenil.
\end{abstract}

Palabras Claves: Redes sociales, Empresas, Posicionamiento, Jóvenes.

\begin{abstract}
:
This study investigates the behavior of young Hondurans from San Pedro Sula in regards to the use of social networks and positioning of companies whose services or products are devoted to it, in order to give these companies information that will improve their access to the youth market. The research findings are the result of a survey applied to three hundred eighty four people between the ages of fifteen and twenty-four years old, during the month of September, with a 0.799 reliability based on Cronbach's alpha. The vast majority of young "sampedranos" use non-traditional electronic media, have internet all the time, use social networks and are willing to receive advertising through them. However, these youths have scarce knowledge of Honduran companies whose services or products are devoted to them and, even lower, of which Companies are interacting with them through social networks. This study provides information that will help the success of advertising by Honduran companies targeting youths.
\end{abstract}

Keywords: Social networks, Companies, Positioning, Youth.

\section{Introducción}

El surgimiento de las redes sociales ha hecho que el mundo se haga cada vez más pequeño, las personas tienen cada día mayores posibilidades de pasar conectados con sus familiares o amigos y encontrar la información que buscan mediante estas redes en poco tiempo y con una facilidad inesperada. Las redes sociales se han convertido en una herramienta muy útil mediante las cuales las empresas pueden estar en contacto son sus

\footnotetext{
${ }^{1}$ Articulo ganador del Segundo Lugar en la categoría de Estudiantes de Pregrado del Concurso de Investigación UNITEC-CEUTEC 2014.

${ }^{2}$ Autor para correspondencia. Email: andreavallecillo@unitec.edu
} 
consumidores, responder inquietudes de estos o simplemente para comunicarles de una nueva promoción o un nuevo producto. Es por eso que hemos realizado este estudio que trata sobre el posicionamiento de las empresas hondureñas en jóvenes sampedranos mediante redes sociales.

El presente estudio abordará temas que van desde cual es el medio electrónico no tradicional que los jóvenes sampedranos utilizan más, hasta cuales son las empresas que ellos conocen cuyos productos o servicios sean dedicados al mercado juvenil y que interactúan mediante redes sociales con ellos.Nuestra investigación es importante porque es clave que las empresas hondureñas sepan llegar con la publicidad indicada a los clientes indicados en el lugar indicado, de esta manera harán que sus estrategias de publicidad sean efectivas y ahorrarán costos. Los resultados están basados en una investigación exploratoria y concluyente, utilizando como herramienta una encuesta aplicada en el mes de septiembre a trescientas ochenta y cuatro personas entre las edades de quince y veinticuatro años.

\subsection{Preguntas de investigación:}

1. ¿Cuál es la red social de mayor uso por los jóvenes?

2. ¿Mediante que dispositivo portátil se conectan más los jóvenes?

3. ¿Cuál es el tiempo de conexión de los jóvenes?

4. ¿Cuáles son los puntos de acceso a internet de los jóvenes?

5. ¿Cuáles son las empresas hondureñas dirigidas a jóvenes que ellos conocen?

6. ¿Mediante qué red social están dispuestos los jóvenes a recibir publicidad de empresas hondureñas?

7. ¿Cómo pueden las empresas hondureñas utilizar las redes sociales para llegar al mercado meta juvenil?

\subsection{Objetivo general:}

El objetivo es triple, (1) Definir las redes sociales con las que los jóvenes sampedranos tienen mayor interacción, (2) Determinar el grado de posicionamiento de las empresas que utilizan estas redes para acceder a los consumidores juveniles, y (3) Entregar a las empresas información sobre uso de las redes sociales para mejorar su acceso al mercado juvenil.

\subsection{Objetivos específicos:}

1. Identificar qué red social es la de mayor interacción en el mercado juvenil, así como el dispositivo portátil de mayor uso.

2. Conocer cuál es el tiempo de conexión de los jóvenes sampedranos, así como también los puntos donde tienen acceso a internet.

3. Mostrar el grado de conocimiento de los jóvenes sampedranos en cuanto a las empresas hondureñas que interactúan electrónicamente con ellos.

4. Analizar mediante qué red social les gustaría a los jóvenes sampedranos recibir publicidad de empresas hondureñas.

5. Determinar cómo las empresas hondureñas pueden hacer uso de las redes sociales como una útil herramienta para estar en contacto con su mercado meta y establecer relaciones redituables con los jóvenes. 


\section{Marco Conceptual y Teórico}

Para uso específico de este informe se utilizan los siguientes términos:

Red social: "Es una estructura social formada por personas o entidades conectadas y unidas entre sí por algún tipo de relación o interés común.” (Ponce, 2012)

Dispositivo portátil: "Los dispositivos portátiles incorporan la unidad del sistema, la unidad de entrada y la unidad de salida en un único paquete liviano.” (Flores, 2012) Según Flores, los dispositivos portátiles, pueden ser transportados por el usuario.

Empresa: "Unidad de organización dedicada a actividades industriales, mercantiles o de prestación de servicios con fines lucrativos.” (Diccionario de la lengua española, 2001)

Juventud: "La Organización Mundial de la Salud (OMS) define al grupo adolescente como la población definida entre los 10 y 19 años, y como jóvenes el grupo comprendido entre 15 y 24 años” (Donas, 1997)

Medio electrónico: "Mecanismo, instalación, equipamiento o sistema que permite producir, almacenar o transmitir documentos, datos e informaciones, incluyendo cualquier red de comunicación abierta o restringida como Internet, telefonía fija y móvil o de otros.” (Derecho.com, s.f.)

Posicionamiento: "Posicionamiento es un término que sirve para describir el lugar o posición que ocupa o puede ocupar un conjunto de imágenes simbólicas en la mente de un determinado sector del público como resultado de un proceso de comunicación persuasiva.” (Figueroa, 1999). Para Figueroa supone la exposición selectiva de un número indeterminado de mensajes de comunicación masiva destinados a un nicho definido dentro de un segmento particular de mercado, con características intergrupales relativamente semejantes en una determinada sociedad.

En países donde la internet tiene bajos niveles de penetración, los anunciantes están cada vez menos dispuestos a probar los medios digitales para la comunicación de sus marcas, y aunque la certeza y comprobación práctica del poder de las redes sociales despierta curiosidad e interés, los detiene el temor de dar a conocer información que haga a sus empresas vulnerables ante las críticas.

Honduras es uno de tales países, con un bajo número de usuarios de la Internet y es por esto que se vuelve interesante estudiar cómo los jóvenes sampedranos interactúan con las redes sociales, cuánto conocen de la publicidad de empresas anunciantes y cuánta y cuál información estarían dispuestos a recibir. Esta información puede interesar a las empresas hondureñas, porque podrían estar ante una nueva herramienta de contacto con su mercado meta.

El mundo digital tiene una importancia creciente en el mercadeo de todos los productos y servicios. Se sabe que el movimiento digital se está consolidando en la región, y que una empresa que no se adapte a esta nueva forma de trabajar, se expone a quedar fuera del mercado.

Según el Informe de la Medición de la Sociedad de la Información 2012 de la Unión Internacional de Telecomunicaciones "el porcentaje de las personas utilizando internet en Honduras en el año 2010 era de aproximadamente 11\%, mientras que el 2011 aumentó a 16\%” (2012). En la Tabla 1 se detalla la información correspondiente a los puntos de acceso a internet durante los años de 2010 y 2011, resaltando que los jóvenes se mantienen conectados con el mundo cibernético mediante teléfonos inteligentes y por computadoras en sus hogares. 
Tabla 1. Puntos de acceso a internet año 2010 y 2011

\begin{tabular}{|l|c|c|}
\hline \multicolumn{1}{|c|}{ Punto de acceso } & $\mathbf{2 0 1 0}$ & $\mathbf{2 0 1 1}$ \\
\hline Hogares con al menos una computadora & $12.9 \%$ & $14 \%$ \\
\hline Hogares con acceso a internet & $6.8 \%$ & $10 \%$ \\
\hline Suscripciones a internet de alta velocidad (banda ancha) & $0 \%$ & $0 \%$ \\
\hline $\begin{array}{l}\text { Suscripciones de internet de banda ancha para dispositivos } \\
\text { móviles }\end{array}$ & $1.3 \%$ & $3.7 \%$ \\
\hline Suscripciones de telefonía celular por cada 100 habitantes & 125.1 & 140 \\
\hline
\end{tabular}

Fuente: Union Internacional de Telecomunicaciones (2012)

En la Tabla 2 se analiza el perfil del usuario de internet en Honduras en el año 2012. Como se conoce que los usuarios de Internet se ubican mayoritariamente en las principales ciudades, y; siendo San Pedro Sula la segunda más poblada, este perfil con seguridad sería similar al de esta ciudad.

Tabla 2. El usuario de internet en Honduras en el año 2012.

\begin{tabular}{|c|c|}
\hline Genero & Porcentaje \\
\hline Hombres & $52.5 \%$ \\
\hline Mujeres & $47.5 \%$ \\
\hline
\end{tabular}

\begin{tabular}{|c|c|}
\hline Nivel socioeconómico & Porcentaje \\
\hline Alto & $30 \%$ \\
\hline Medio & $46 \%$ \\
\hline Bajo & $22 \%$ \\
\hline Muy bajo & $2 \%$ \\
\hline
\end{tabular}

\begin{tabular}{|c|c|}
\hline Edad & Porcentaje \\
\hline 12 a 17 años & $25 \%$ \\
\hline 18 a 24 años & $27 \%$ \\
\hline 25 a 39 años & $28 \%$ \\
\hline 40 años y más & $20 \%$ \\
\hline
\end{tabular}

\begin{tabular}{|c|c|}
\hline Lugar de residencia & Porcentaje \\
\hline Zona urbana & $86 \%$ \\
\hline Zona rural & $14 \%$ \\
\hline
\end{tabular}

\begin{tabular}{|c|c|}
\hline $\begin{array}{c}\text { Tipo de información que } \\
\text { consume vía internet }\end{array}$ & Porcentaje \\
\hline Educación & $54.4 \%$ \\
\hline Entretenimiento & $53.2 \%$ \\
\hline Información y noticias & $49.7 \%$ \\
\hline Comunicación & $47.9 \%$ \\
\hline
\end{tabular}

\begin{tabular}{|c|c|}
\hline $\begin{array}{c}\text { Frecuencia de uso de } \\
\text { internet }\end{array}$ & Porcentaje \\
\hline Una vez al día & $45.2 \%$ \\
\hline Una vez por semana & $42.1 \%$ \\
\hline Una vez al mes & $11 \%$ \\
\hline
\end{tabular}

Fuente: INE (s.f.) 
El auge de las redes sociales ha provocado el incremento del internet. En la Tabla 3 se ve reflejado que Facebook fue la red mas visitada por los usuarios en Honduras. En enero del 2012 registró 690 millones de visitas.

Tabla 3. Redes sociales más usadas durante el 2012

\begin{tabular}{|c|c|}
\hline Red social & Visitas en millones \\
\hline Facebook & 690 \\
\hline Windows live profile & 170 \\
\hline Twitter & 110 \\
\hline Badoo & 69 \\
\hline Sonico & 40 \\
\hline Linkedln & 30 \\
\hline Hi5 & 29 \\
\hline
\end{tabular}

Fuente: iLifebelt (2012)

Este dato también esta respaldado por el recuento del top 10 de los sitios más visitados en Honduras en 2012 (Alexa, 2012), que se detalla de la siguiente manera:

1. Facebook

2. Google Honduras

3. YouTube

4. Yahoo!

5. Google
6. Windows live

7. Babylon

8. Wikipedia

9. BlogSpot

10. Twitter

La lista anterior es un indicador de cómo y en qué los usuarios en Honduras utilizan la red cibernética. Se observa el claro predominio de las redes sociales, entre las que destaca Facebook, Google Honduras, YouTube y los Blogs. Facebook se ha convertido en un grande en la actualidad mediante la dinámica de su página social, la cual lo ha llevado al éxito. Los hondureños utilizan google para la búsqueda de información, ya sea para elaborar trabajos o encontrar referencia de datos relevantes. En el top 10 se observa que el consumidor hondureño busca entretenerse con las páginas sociales que internet brinda hoy en día, como las redes sociales y los blogs. YouTube se encuentra en la posición número tres, lo que indica que los habitantes de Honduras optan por el entretenimiento audiovisual, un dato importante para que las empresas y agencias de publicidad puedan utilizar este medio para su promoción.

De igual forma, se conoce que cada red social tiene un tipo de comportamiento diferente, por ejemplo, para lograr efectividad en Facebook, es necesario definir la audiencia y sus comportamientos en el medio, horarios de uso, tipo de contenido que consume, forma que se conecta, así como tener claro lo que se desea lograr con la presencia en esta red social. Las reglas de oro del medio son la interacción con el usuario, la atención de la página y la humanización del medio.

Con respecto a twitter, se debe tomar en cuenta que el usuario tiende a ser más experimentado en redes sociales, razona más puntos de conexión y se toma más libertad en emitir juicios tanto positivos como negativos.

\section{Metodología y Hallazgos}

Los datos de este estudio proceden de una encuesta a una muestra de 384 jóvenes sampedranos entre las edades de 15 a 24 años. Se estima que este tamaño muestral produce un nivel de confianza de los datos de $95 \%$. 
Se llegó a la conclusión que la mayoría de los jóvenes sampedranos utilizan medios electrónicos no tradicionales, tienen acceso a internet todo el tiempo, utilizan redes sociales y están dispuestos a recibir publicidad mediante ellas. Sin embargo, es muy escaso el conocimiento de los jóvenes acerca de cuáles son las empresas hondureñas cuyos servicios o productos son dedicados a ellos, y es mucho más bajo el conocimiento de cuáles son las empresas hondureñas que están interactuando mediante redes sociales con ellos. Algunos de los hallazgos se detallan a continuación.

De los 384 jóvenes encuestados, 99.5\% declararon utilizar algún medio electrónico no tradicional. De estos usuarios, 82\% utiliza frecuentemente un teléfono inteligente, 11\% computadoras y $7 \%$ acude a las tabletas.

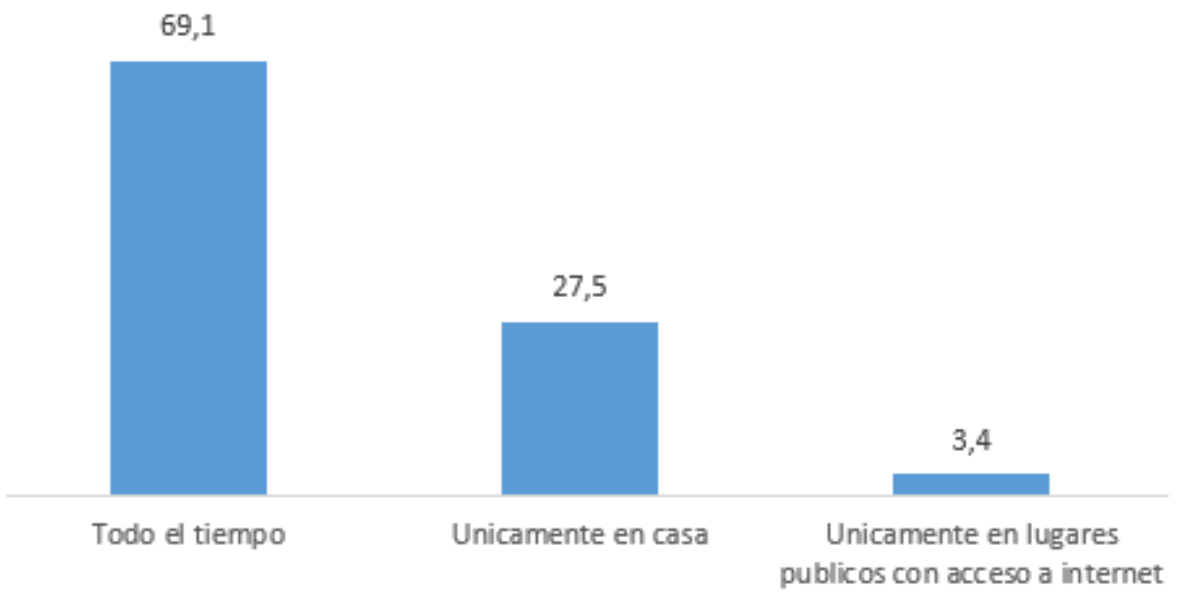

Figura 1. Momentos de acceso a internet

Como se puede observar en la Figura 1, el 69.1\% de los encuestados tiene acceso a internet todo el tiempo, el 27.5\% únicamente en casa y un 3.4\% tiene acceso a internet únicamente en lugares públicos.

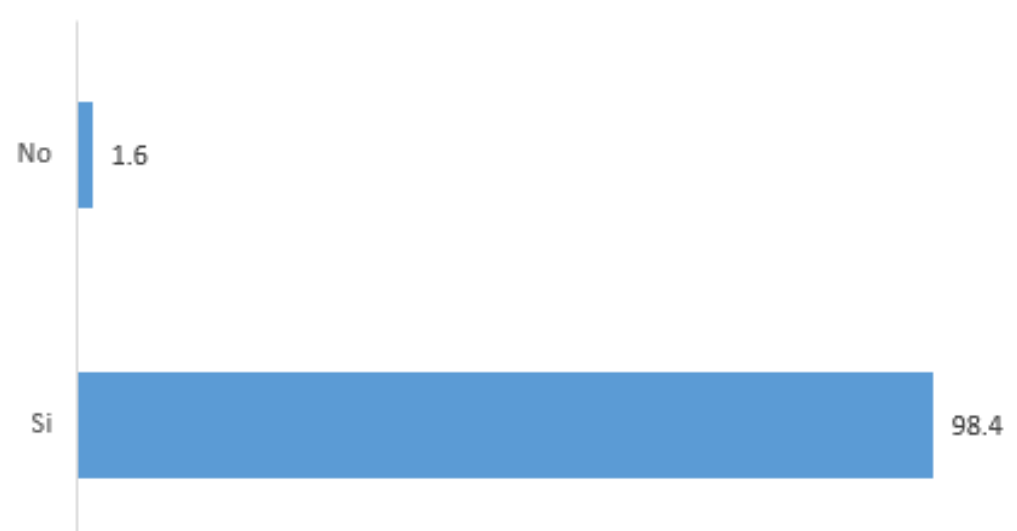

Figura 2. Uso de red social

Como se muestra en la Figura 2, 376 personas; es decir el 98.4\% de las 382 que si utilizan un medio electrónico no tradicional utilizan alguna red social, únicamente el 1.6\%, que representa a 6 personas no hacen uso de ellas. 
- de 1 a 3 horas $=$ de 4 a 6 horas $=$ de 7 a 10 horas $=$ de 11 horas en adelante

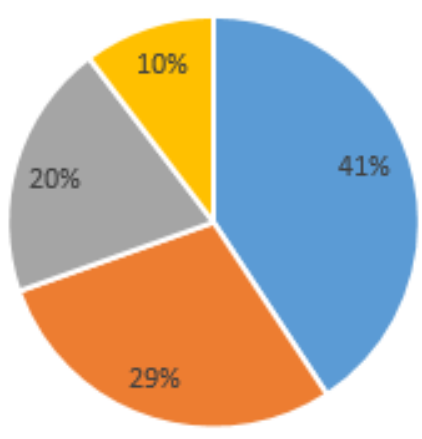

Figura 3. Promedio de uso de las redes sociales

En la Figura 3 se aprecia que del 100\% que si utilizan redes sociales, el $41 \%$ utilizan las redes sociales de 1 a 3 horas, el 29\% de 4 a 6 horas, el 20\% de 7 a 10 horas y el $10 \%$ de 11 horas en adelante.

Tabla 4. Frecuencia de uso de las redes sociales

\begin{tabular}{|c|c|c|c|c|c|}
\hline Red social & $\mathbf{1}$ & $\mathbf{2}$ & $\mathbf{3}$ & $\mathbf{4}$ & $\mathbf{5}$ \\
\hline Facebook & $\mathbf{2 8 . 7}$ & 18.1 & 23.4 & 14.9 & 14.9 \\
\hline Whatsapp & $\mathbf{6 0 . 2}$ & 10.3 & 4.5 & 4.2 & 20.7 \\
\hline Twitter & 16.6 & 9.9 & 14.7 & 11 & $\mathbf{4 7 . 7}$ \\
\hline Instagram & 20.4 & 20.1 & 13.4 & 12.9 & $\mathbf{3 3 . 2}$ \\
\hline Line & 15.1 & 5.9 & 11.6 & 9.2 & $\mathbf{5 8 . 1}$ \\
\hline Snapchat & 20.4 & 8.9 & 11.8 & 10.5 & $\mathbf{4 8 . 4}$ \\
\hline Skype & 14.1 & 8.4 & 15.4 & 13.3 & $\mathbf{4 8 . 4}$ \\
\hline Correo electrónico & 23.4 & 21.5 & 15.7 & 11.2 & $\mathbf{2 8 . 2}$ \\
\hline
\end{tabular}

Siendo 1 la calificación de la red social más utilizada y 5 la de la menos utilizada, observamos que Facebook y Whatsapp fueron calificados como la red social más utilizada con $28.7 \%$ y $60.2 \%$ respectivamente. Las otras redes sociales como Twitter, Instagram, Line, Snapchat, Skype y el correo electrónico fueron calificados por la mayoría como las menos utilizadas como se puede apreciar en la Tabla 4.

Emocionales accion $\mathbf{m}$ Graciosos a

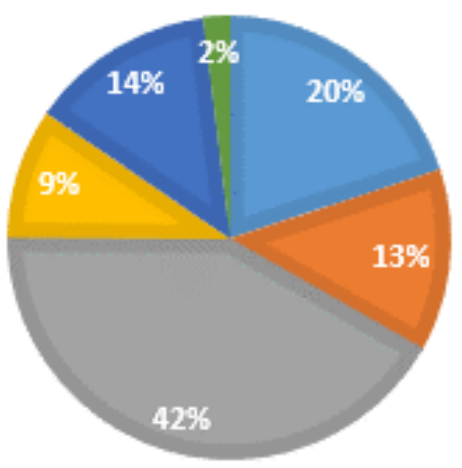

Figura 4. Tipos de anuncios de preferencia 
En la Figura 4 se puede apreciar que $42 \%$ de los encuestados prefieren los anuncios graciosos, el 20\% los emocionales, el 14\% con aparición de famosos, 13\% de acción, 9\% de suspenso y solo el $2 \%$ prefieren otros tipos de anuncios.

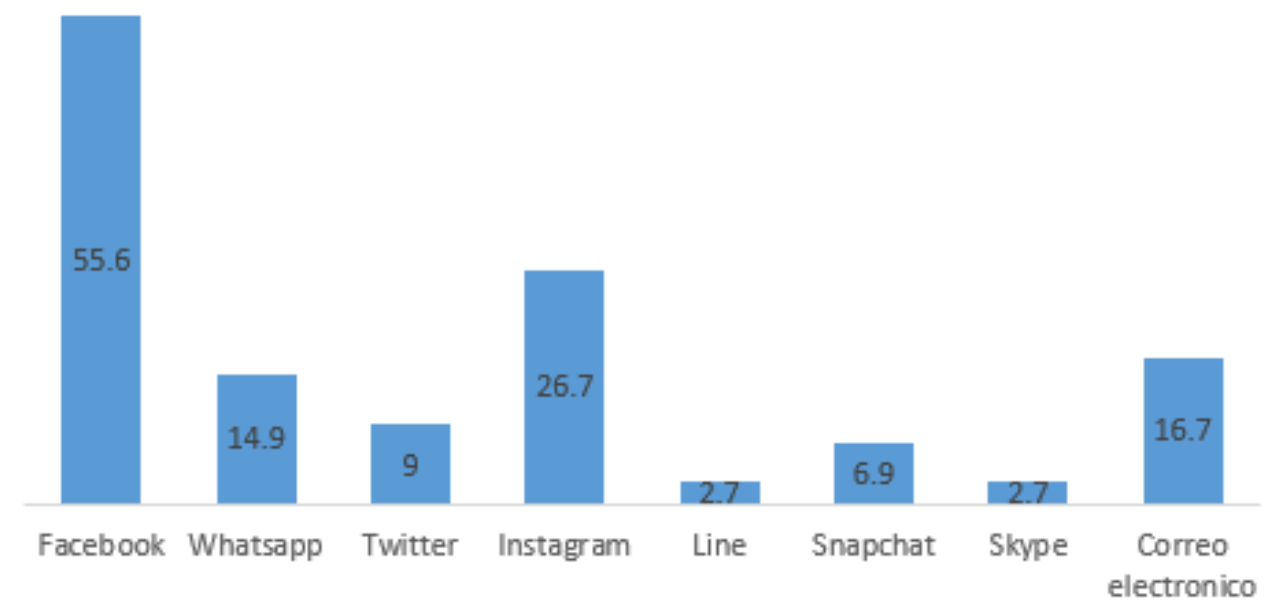

Figura 5. Red social de preferencia para recibir publicidad

Como se aprecia en la Figura 5, las redes sociales que destacan por ser las que las personas prefieren para recibir publicidad son: Facebook con un 55.6\%, Instagram con un 26.7\%, el correo electrónico con un 16.7\% y Whatsapp con un 14.9\%.

Tabla 5. Empresas hondureñas que conoce que tienen servicios o productos dedicados a jóvenes.

\begin{tabular}{|c|c|c|}
\hline Empresa & Frecuencia & Porcentaje \\
\hline Ninguna & 1407 & 62.37 \\
\hline Otras & 437 & 19.37 \\
\hline Carrión & 68 & 3.01 \\
\hline Baleadas Express & 51 & 2.26 \\
\hline Espresso Americano & 44 & 1.95 \\
\hline The heels & 43 & 1.91 \\
\hline Pollo el Hondureño & 43 & 1.91 \\
\hline Diunsa & 42 & 1.86 \\
\hline Cervecería Hondureña & 38 & 1.68 \\
\hline Wingers & 32 & 1.42 \\
\hline Galeano & 30 & 1.33 \\
\hline Útiles de Honduras & 21 & 0.93 \\
\hline Total & $\mathbf{2 2 5 6}$ & $\mathbf{1 0 0 . 0 0}$ \\
\hline
\end{tabular}

La Tabla 5 muestra que de los 376 encuestados que si utilizan medios electrónicos no tradicionales y redes sociales, el $62.37 \%$ no conoce ninguna empresa hondureña cuyos servicios sean dedicados a jóvenes, del restante; el 19.37\% representa otras empresas que apenas recibieron de una a cinco menciones en el estudio. Las empresas que tuvieron más menciones fueron en orden de mayor a menor: Carrión, Baleadas Express, Espresso Americano, The heels, Pollo el Hondureño, Diunsa, Cervecería Hondureña, Wingers, Galeano y Útiles de Honduras. 

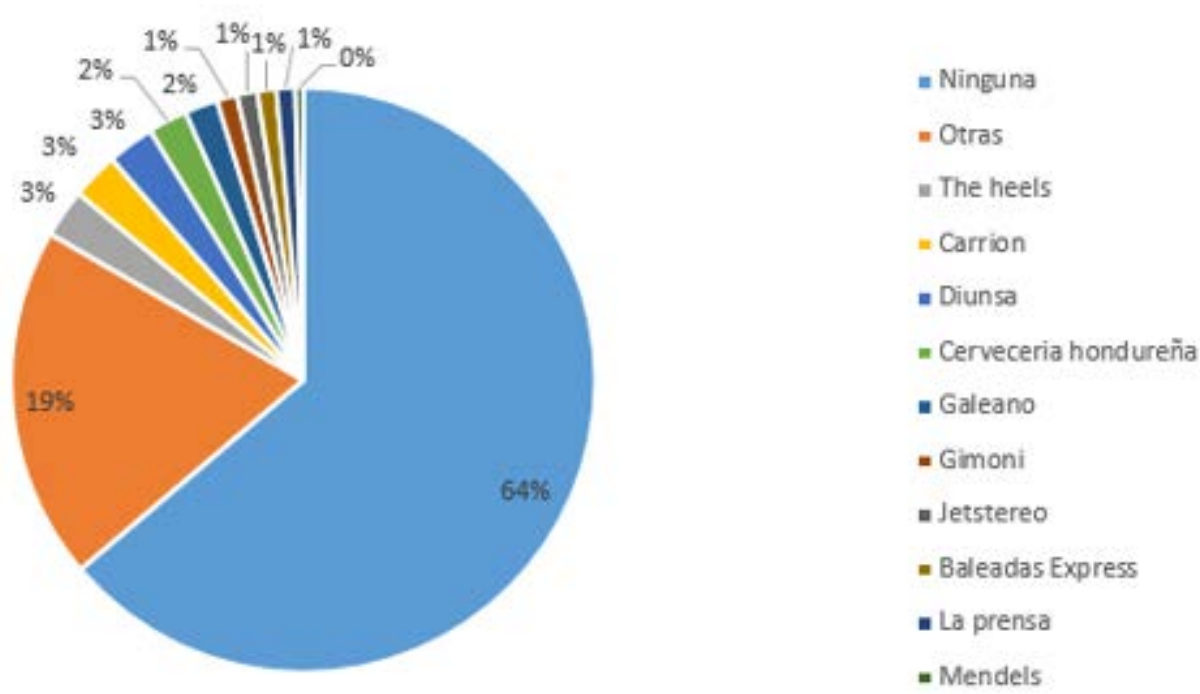

Figura 6. Empresas hondureñas que conoce cuyos servicios o productos son dedicados a jóvenes y que interactúan mediante redes sociales con los consumidores.

Como refleja la Figura 6, de los 376 encuestados, el 64\% no conoce ninguna empresa hondureña cuyos servicios sean dedicados a jóvenes y que interactúan mediante redes sociales, del restante el 19\% representa otras empresas que apenas recibieron de una a cinco menciones en el estudio. Las empresas que tuvieron más menciones fueron en orden de mayor a menor: The heels, Carrión, Diunsa, Cervecería Hondureña, Galeano, Gimoni, Jetstereo, Baleadas Express, La Prensa y Mendels.

\section{Conclusiones}

El surgimiento de las redes sociales ha hecho que el mundo se haga cada vez más pequeño, las redes sociales se han convertido en una herramienta muy útil mediante las cuales las empresas pueden estar en contacto con sus consumidores e interactuar con ellos.

Los resultados de este estudio indican que los jóvenes utilizan medios electrónicos no tradicionales. La mayoría utiliza teléfonos inteligentes y tienen acceso a internet todo el tiempo. Dichos jóvenes hacen uso de alguna red social en un promedio de 1 a 3 horas y Facebook y Whatsapp son las más utilizadas.

Respecto a publicidad, la mayoría de los encuestados prefieren anuncios graciosos y, las redes sociales que prefieren para recibir publicidad son Facebook, Instagram y el correo electrónico. Referente al posicionamiento de empresas hondureñas cuyos servicios van orientados al mercado juvenil, la mayoría de los jóvenes no conocen empresas cuyos productos o servicios van orientados a ellos y es aún menor el conocimiento de cuales de estas empresas interactúan mediante redes sociales con ellos.

Es importante que las empresas hondureñas evalúen cual es el medio correcto para llegar a su público meta, para de esta forma, asegurarse que sus estrategias de publicidad sean efectivas. Conocer los medios adecuados y los horarios con más tráfico en las redes sociales ayudará a poder maximizar el posicionamiento de las compañías hondureñas. Las empresas hondureñas tienen el reto de mejorar la comunicación con sus 
consumidores, ir más allá de los medios tradicionales e incursionar en nuevas plataformas como las redes sociales y e-business, que les permitirán comunicarse de una manera más efectiva con los clientes.

\section{Bibliografía}

(s.f.).Retrieved from Derecho.com:

http://www.derecho.com/c/Medio_electr\%C3\%B3nico

(s.f.). In T. Kinnear, \& J. Taylor, Investigación de Mercados (p. 301). Mc Graw Hill.

(2001). En D. d. española, Diccionario de la lengua española (pág. 837). España:

Espasa Calpe.

Alexa. (2012, Octubre 28 Octubre 2012). Retrieved from Alexa: www.alexa.com

Benitez, J. (2012). El pajarito comunicador. Advertising Age, 29.

Cubías, C. (2012). El poder de conocer al usuario. Advertising Age, 28.

Donas, S. M. (1997). El concepto de juventud. 3.

Figueroa, R. (1999). Como hacer publicidad. México: Addison Wesley Longman de México.

Flores, J. (2012). Tecnologías de la información y comunicación. México.

Gomez, R. R. (2013). La promoción: resultados y mejor forma de implementación. Advertising Age, 24.

iLifebelt, G. (2012). Estudio de las redes sociales en Centroamérica.

INE. (s.f.). Comisión nacional de telecomunicaciones Conatel Honduras.

Informe de medición de la sociedad de la informacion 2012 de la unión internaciontal de telecomunicaciones. (2012). 36.

Juan Trespalacios Gutiérrez, R. V. (2005). Investigación de Mercados. International Thomson Editores.

Malhotra, N. (2008). Investigación de mercados. México: Pearson Educación.

Ortega, K. (2011). El movimiento digital se consolida en la region. Advertising Age, 4.

Ponce, I. (2012, Abril 17). Observatorio tecnológico. Retrieved from Observatorio tecnológico: http://recursostic.educacion.es/observatorio/web/es/internet/web20/1043-redes-sociales?start=1 
"LA REVISTA INNOVARE NO SE HACE RESPONSABLE EN NINGÚN CASO DE LOS CONTENIDOS, DATOS, CONCLUSIONES U OPINIONES VERTIDAS EN LOS ARTÍCULOS PUBLICADOS, SIENDO ESTA RESPOSABILIDAD EXCLUSIVA DEL (DE LOS) AUTOR (AUTORES)” 